\title{
ROLE OF TRANSABDOMINAL ULTRASONOGRAPHY IN THE EVALUATION OF SUSPECTED ECTOPIC PREGNANCY
}

\author{
NAHAR MN ${ }^{1}$, SATTAR A $^{2}$, ARA H $^{3}$, RABBI AF ${ }^{4}$, SHIRIN M$^{5}$, KUMU FK $^{6}$
}

\begin{abstract}
The study was carried out to assess the diagnostic accuracy of transabdominal ultrasonography in the evaluation of clinically suspected ectopic pregnancy patients. This cross sectional study was carried out in the department of Radiology and Imaging, Dhaka Medical College Hospital, Dhaka, from July 2006 to June 2008. 50 patients were selected purposively and transabdominal ultrasonography was done in each patient by $3.5 \mathrm{MHz}$ frequency curvilinearprobe. Findings of transabdominal ultrasonography were then compared with the peroperative findings. Transabdominal ultrasonography in the diagnosis of ectopic pregnancy showed a sensitivity of $79.5 \%$, specificity of $83.3 \%$, and accuracy of $80 \%$. Positive predictive value was $97.2 \%$ and negativepredictive value was $55.6 \%$. This study shows thattransabdominal ultrasonographyis an effective modality in the evaluation of ectopic pregnancy and should be used as a routine first-step procedure in patients of suspected ectopic pregnancy.
\end{abstract}

Key words: Ectopic pregnancy, ultrasonography.

J Dhaka Med Coll. 2013; 22(2) : 167-172.

\section{Introduction:}

Ectopic pregnancy represents implantation of the fertilized ovum outside the uterine cavity. In $95 \%$ of the cases it is localized in the fallopian tube $(95 \%)$ but abdominal cavity, ovary, intraligamentous location, cornual, intramural or cervical sites are not unusual ${ }^{1}$.Ectopic pregnancy during the first trimester is the leading cause of pregnancyrelated death in the USA ${ }^{2}$. Not only women die from this disease but approximately two-thirds of those who survive will never subsequently bear a living child and at least one out of ten will have a second ectopic gestation. The incidence rate has risen dramatically during the past 30 years $^{3}$ as a consequence of injudicious antibiotic therapy, reconstructive tubal surgery, and the use of intrauterine contraceptive devices. The symptoms and signs are similar to those of many other gynaecological disorders and clinical suspicion will be high in any woman of child-bearing age who presents with lower abdominal pain and abnormal vaginal bleeding. Ectopic pregnancy is suspected by clinicians 10 times more often than it occurs ${ }^{4}$ and radiology departments providing an ultrasound service for gynaecological patients will receive many referrals for a suspected ectopic pregnancy. Historically, ectopic pregnancies were diagnosed and managed surgically in symptomatic women, often presenting with the classic triad of symptoms: pain, vaginal bleeding and a history of amenorrhoea. However, now with advances in the use of diagnostic ultrasound and the rapid

1. Dr. Mst. Nazmun Nahar, Assistant Professor, Department of Radiology and Imaging, Dhaka Medical College, Dhaka.

2. Dr. Asifa Sattar, Assistant Professor, Department of Radiology and Imaging, Sir Salimullah Medical College, Dhaka.

3. Dr. Husne Ara, Assistant Professor, Department of Radiology and Imaging, Dhaka Medical College, Dhaka.

4. Dr. Akanda Fazle Rabbi, Assistant Professor, Department of Radiology and Imaging, Dhaka Medical College, Dhaka.

5. Dr. Mahbuba Shirin, Assistant Professor, Department of Radiology and Imaging, Bangabandhu Sheikh Mujib Medical University (BSMMU), Dhaka.

6. Dr. Farhana Kamal Kumu, Indoor Medical Officer, Department of Radiology and Imaging, Dhaka Medical College Hospital, Dhaka

Correspondence : Dr. Mst. Nazmun Nahar, Assistant Professor, Department of Radiology and Imaging, Dhaka Medical College, Dhaka. 
immunoassay of serum human chorionic gonadotrophin, it is possible to diagnose an ectopic pregnancy at an earlier stage prior to treatment, and even manage them nonsurgically using either an expectant or medical approach.. Diagnostic ultrasound played an important role in the investigation of the patient with a suspected ectopic pregnancy since 1965, when Professor Ian Donald suggested its important role was to exclude the condition by demonstrating an intrauterine pregnancy. Early reports suggest that the diagnostic accuracy of ultrasound may be greater using transvaginal probes ${ }^{5}$. But it is not yet in widespread use and available in few higher centre of our country. High resolution abdominal ultrasonography helps to make early diagnosis of intrauterine gestation. The confirmation of an intrauterine pregnancy is sufficient to exclude ectopic gestation because the incidence of combined intrauterine pregnancy and ectopic pregnancy (heterotopic pregnancy) is approximately 1 in $30,000^{6}$.This study was carried out to assess the efficacy of transabdominal ultrasonography in the detection of ectopic pregnancy.

\section{Methods:}

This cross sectional study was carried out in department of Radiology and Imaging with collaboration of department of Obstetrics and Gynaecology, Dhaka Medical College Hospital, Dhaka, from July 2006 to June 2008 on the patients with clinical suspicion of ectopic pregnancy. 50 patients were selected purposively meeting the following inclusion and exclusion criteria. Clinically suspected and incidentally detected cases of ectopic pregnancy were included in the study and those having normal or abnormal intrauterine pregnancy and those not admitted in this hospital and those with unavailability of peroperative findings were excluded. After giving a brief explanation of the procedure all underwent transabdominal ultrasonography performed by curvilinear transducer of $3.5 \mathrm{MHz}$ of Esaote Biomedia AU530 ultrasound machine and Fukuda desnshi FF sonic- 400. Imaging of the uterus and adnexa were performed in both sagittal and transverse planes. The urinary bladder was full. During ultrasound examination, special note was made on presence of adnexal mass, peritoneal collection in Cul-De-Sac and hepatorenal pouch, extrauterine gestational sac and embryo with cardiac activity. The criteria for diagnosis of ectopic pregnancy included an extrauterine gestational sac containing a foetus or a foetal pole or an empty extrauterine sac. Solid or complex adnexal mass, peritoneal collection, peudogestational sacs were considered suggestive and correlated with pregnancy test (âhCG in urine and serum). All patients underwent surgery and peroperative findings were collected. Surgical diagnosis of ectopic pregnancy was made by visualization of foetal parts, gestational sac, haemoperitoneum, tubal staus and in doubtful cases specimen was sent for histopathological examination. Ethical clearance was taken from the institutional ethical committee of Dhaka Medical College.

After collection of the data sensitivity, specificity, accuracy, positive and negative predictive values of transabdominal ultrasonography in the diagnosis of ectopic pregnancy were calculated by appropriate statistical formula. In every case the ultrasonographic findings were compared with respective surgical findings.

\section{Results:}

Initially 178 patients of clinically suspected ectopic pregnancies reporting to Radiology and imaging department of Dhaka Medical College, Dhaka were enrolled in this study. After analyzing selection criteria 50 patients were included in final analysis. Ultrasonographic and surgical diagnoses were made according to the criterias mentioned in materials and methods.

Age of the study patients ranged from 18 to 36 years with largest number of patients in the 21-25 years group.

Abdominal pain was the most common presenting features occurring in $90 \%$ cases. The pain varied in severity from a mild ache or cramp to a sudden, severe, sharp agonizing pain, initially located in lower abdomen or one side but gradually spreading all over the abdomen. A short period of amenorrhea ranging 
from 5-8 weeks was present in $76 \%$ cases.Pervaginal bleeding ranging from slight spotting to moderate in amount was present in $72 \%$ case, $28 \%$ cases presented with a palpable lower abdominal mass.

Pelvic inflammatory disease recorded in 32 cases was the most common risk factor in ectopic pregnancy followed by history of previous ectopic pregnancy in $10 \%$. History of appendicitis $6 \%$, history of tubal surgery $2 \%$ and equal percentage had history of salpingits. There was no risk factor in $46 \%$ cases.

Gravida of the study patients ranged from 1 to 7. It was evident that the preponderance of ectopic pregnancies was highest in fourth gravida.

The most commonly observed ultrasonographic abnormality was an adnexal mass $30(60 \%)$ followed by peritoneal collection $28(56 \%)$ and enlarged uterus 24(48\%). A gestational sac containing foetal pole with heart motion which is a diagnostic finding of ectopic pregnancy was present in $3(6 \%)$ cases. Gestational sac with foetal pole without heart motion was present in $4(8 \%)$ cases. Gestational sac without embryo or yolk sac was present in $3(6 \%)$ cases. No pseudosac could be identified, although in $10 \%$ cases nonspecific internal echoes were identified. (Table- I).

Table I: Ultrasonographic findings of the patients $(N=50)$

\begin{tabular}{lcc}
\hline Ultrasonographic findings & Frequency & Percent \\
\hline Uterine & 24 & 48.0 \\
Enlarged uterus & 5 & 10.0 \\
Non specific echoes & 0 & 0 \\
Pseudosac & & \\
Adnexal abnormality & 2 & 4.0 \\
Gestational sac with living embryo & 4 & 8.0 \\
Gestational sac with foetal pole & 3 & 6.0 \\
Gestational sac without embryo & 3 & \\
or yolk sac & 30 & 60.0 \\
Non specific adnexal mass & 5 & 10.0 \\
Dilated fallopian tube & & \\
Peritoneal & 28 & 56.0 \\
Fluid & 1 & 2.0 \\
Gestational sac with living & & \\
embryo & & \\
\hline
\end{tabular}

*Multiple responses
Size of adnexal masses of ectopic pregnancy varied from $2-6 \mathrm{~cm}$ with the highest number in $4-5 \mathrm{~cm}$ group $12(40 \%) .20$ cases of adnexal masses showed mixed echo pattern , 5 hypoechoic and 3 hyperechoic pattern. In 20 $(66.7 \%)$ cases adnexal masses were noted on right side and $10(33.3 \%)$ cases were noted on left side.

Out of 10 ectopic gestational sacs 8 were located in adnexal region. 1 gestational sac with live foetus located in peritoneal cavity was diagnosed as intraabdominal pregnancy and in another case gestational sac with live embryo located eccentrically within uterus surrounded by a thin layer of myometrium was diagnosed as cornual pregnancy.

Peritoneal collection was detected in $28(56 \%)$ cases. Collection was moderate to severe in most cases and mild in 2 cases. No peritoneal fluid was noted in 22 cases .Peritoneal fluid was echogenic in 17 (34\%) cases and anechoic in $11(22 \%)$ cases.

$27(54 \%)$ cases were diagnosed as ruptured ectopic pregnancy by ultrasound along with clinical history and pregnancy test report (in available cases). 7 (14\%) cases were diagnosed as unruptured ectopic pregnancy, $1(2 \%)$ case intraabdominal pregnancy, $1(2 \%)$ cornual pregnancy. $10(20 \%)$ cases were diagnosed as pelvic inflammatory disease, $1(2 \%)$ twisted ovarian cyst, 1 (2\%) appendicitisand $2(4 \%)$ cases were reported as normal sonographic findings (Table- II).

Table II

Ultrasonographic diagnosis of the study patients

\begin{tabular}{lcc}
\hline Ultrasonographic diagnosis & Frequency & Percent \\
\hline Ruptured ectopic pregnancy & 27 & 54.0 \\
Un ruptured ectopic pregnancy & 7 & 14.0 \\
Intra-abdominal pregnancy & 1 & 2.0 \\
Cornual pregnancy & 1 & 2.0 \\
Pelvic inflammatory disease & 10 & 20.0 \\
Twisted ovarian cyst & 1 & 2.0 \\
Normal study & 2 & 4.0 \\
Appendicitis & 1 & 2.0 \\
\hline Total & 50 & 100.0 \\
\hline
\end{tabular}

Surgical exploration revealed highest percentage of patients diagnosed as ruptured ectopic pregnancy 30 (60\%) followed by 
unruptured ectopic pregnancy $6(12 \%)$, old ectopic pregnancy $4(8 \%)$, tubal abortion $2(4 \%)$, pelvic inflammatory disease $4(8 \%)$, twisted ovarian cyst $1(2 \%)$, corpus luteual cyst $1(2 \%)$, intraabdominal pregnancy $1(2 \%)$ and cornual pregnancy $1(2 \%)$ (Table- III).

Table III

Surgical diagnosis of the study patients

\begin{tabular}{lcc}
\hline Surgical diagnosis & Frequency & Percent \\
\hline Ruptured ectopic pregnancy & 30 & 60.0 \\
Unruptured ectopic pregnancy & 6 & 12.0 \\
Old ectopic pregnancy & 4 & 8.0 \\
Tubal abortion & 2 & 4.0 \\
Intra-abdominal pregnancy & 1 & 2.0 \\
Cornual pregnancy & 1 & 2.0 \\
Pelvic inflammatory disease & 4 & 8.0 \\
Twisted ovarian cyst & 1 & 2.0 \\
Corpus luteal cyst & 1 & 2.0 \\
\hline Total & 50 & 100.0 \\
\hline
\end{tabular}

Sensitivity and specificity analysis of ultrasonographic diagnosis and surgical diagnosis revealed that the sensitivity, specificity, positive predictive and negative predictive value and accuracy for ultrasonographic diagnosis were $79.5 \%, 83.3 \%$, $97.2 \%, 55.6 \%$ and $80.0 \%$ respectively (Table- IV).

Table IV

Sensitivity and specificity analysis of ultrasonographic diagnosis correlated with surgical findings

\begin{tabular}{lccc}
\hline Ultrasonographic & \multicolumn{2}{c}{ Surgical diagnosis } & Total \\
diagnosis & Positive & Negative & \\
\hline Positive & $35(79.5)$ & $1(16.7)$ & $36(72.0)$ \\
Negative & $9(20.5)$ & $5(83.3)$ & $14(28.0)$ \\
\hline Total & $44(100.0)$ & $6(100.0)$ & $50(100.0)$ \\
\hline
\end{tabular}

\section{Discussion:}

Initially 178 patients of clinically suspected ectopic pregnancies were enrolled in this study. After analyzing selection criteria 50 percent were included in the final analysis and only
44 of them had proven ectopic gestation. This reflects a high degree of clinical suspicion and referral to radiology department. In 6 cases the final diagnoses were pelvic inflammatory disease and complicated ovarian cyst. The age range of the present study was 18 to 36 years with largest number of patients in the 21-25 years age group. An evaluation of the age distribution substantiates the fact that a woman who is capable of conceiving is capable of having a pregnancy in a location other than the uterine cavity. The most common symptoms presented by the patients were abdominal pain 45 (90\%), Amenorrhea 38 (76\%) per vaginal bleeding $36(72 \%)$ and lower abdominal mass occurred in 14 (28\%) of ectopic pregnancies.

Regarding risk factors in this study 16 (32\%) had history of pelvic inflammatory disease followed by $5(10 \%)$ prior ectopic pregnancy, appendicitis $3(6 \%)$, salpingitis $2(4 \%)$, tubal surgery $1(2 \%) .23$ (46\%) had no risk factor.

Sonographic evaluation of women suspected of having an ectopic pregnancy requires correct interpretation of both intrauterine and extrauterine findings. One patient may have more than one finding. Presence of intrauterine pregnancy virtually excludes an ectopic pregnancy as the incidence of concomitant intrauterine pregnancy and ectopic pregnancy is approximately 1 in $30,000^{6}$. An intrauterine gestation sac with a live fetus is positive proof of an intrauterine pregnancy, but in the majority of patients the gestational age will only be 5- 6 weeks and the sac will be empty. Differentiation between a true gestation sac and a pseudosac may be difficult. Using the criteria of a double decidual sign and eccentric placement of the sac Chambers ${ }^{7}$ identified intrauterine pregnancies correctly. However, in present study no intrauterine gestational sac could be identified, although in $10 \%$ cases nonspecific internal uterine echoes were identified. These intrauterine echoes are probably due to hyperplastic endometrium and blood within the endometrial cavity. According to the modified Kobayashi criteria for ultrasonic diagnosis of ectopic pregnancy uterine enlargement is 
present in $62 \%$ of cases of ectopic pregnancy. In present study uterine enlargement (bulky uterus) was present in $24(48 \%)$ cases.

The most commonly observed ultrasonographic abnormality was an adnexal mass 30 (60\%). The masses were $2-6 \mathrm{~cm}$ in diameter. In the majority the mass was complex with both solid and cystic components. Because an adnexal mass can be observed by abdominal sonography in different conditions (e.g. endometriosis, abscesses) ultrasonography is considered diagnostic of an ectopic pregnancy only if an ectopic gestational sac is visible outside the uterus, with foetal cardiac pulsation demonstratable within. We found ectopic gestational sac in $10(20 \%)$ cases. Among them foetal pole was detected in $4(8 \%)$ cases and gestational sac with living embryo in $2(4 \%)$ cases. Gesstational sac without foetal pole or emryo representing tubal ring sign was present in $3(6 \%)$ cases. $1(2 \%)$ abdominal pregnancy was diagnosed by presence of gestational sac with live foetus in the peritoneal cavity. The observation of a very eccentrically located gestational sac with embryo within the uterus suggested the diagnosis of a cornual pregnancy. Cacciatore et $\mathrm{al}^{8}$ found ectopic gestational sac in $44 \%$ cases and no ectopic living embryo. Lawson ${ }^{9}$ found extrauterine gestational sac in $15 \%$ cases and no ectopic living embryo.

In the absence of specific evidence of an extrauterine gestation (gestational sac with or without an embryo) identification of fluid in the cul-de-sac is an important supportive evidence of ectopic pregnancy. Previous similar studies ${ }^{10-12}$ have reported the presence of free fluid in $40-83 \%$ of patients with ectopic pregnancies. These results are consistent with present study that demonstrated the presence of free fluid in $28(56 \%)$ of cases. Fluid was moderate to larger in amount and echogenic in $17(34 \%)$ and anechoic in $11(22 \%)$ cases. Fluid was absent in 22 (44\%) cases.

Ultrasonographic diagnosis revealed that highest percentage of patients diagnosed as ruptured ectopic pregnancy 27 (54\%) followed by pelvic inflammatory disease 10 (20\%), unruptuered ectopic pregnancy 7 (14\%), intraabdominal pregnancy $1(2 \%)$, cornual pregnancy $1(2 \%)$, twisted ovarian cyst $1(2 \%)$, appendicitis $1(2 \%)$ and $2(4 \%)$ cases were reported as normal sonographic findings.

Surgical exploration revealed that highest percentage diagnosed as ruptured ectopic 30 $(60 \%)$, unruptured ectopic $6(12 \%)$, old ectopic $4(8 \%)$, tubal abortion $2(4 \%)$, pelvic inflammatory disease $4(8 \%)$, intraabdominal pregnancy $1(2 \%)$, cornual pregnancy $1(2 \%)$, twisted ovarian cyst $1(2 \%)$, ruptured corpus luteal cyst $1(2 \%)$.

Analysis of the 9 cases with false negative ultrasonographic diagnosis of ectopic pregnancy revealed that adnexal masses representing the ectopic pregnancy were seen in 7 cases but were misinterpreted as bowel loops or inflammatory disease due to atypical presentation and negative pregnancy test report. In 2 cases no sonographic evidence of ectopic pregnancy was detected and reported as normal findings. But as there was history of prior ectopic pregnancy and serum âhCG was raised. Laparotomy followed by laparoscopy was done and tubal pregnancy was detected. A strong clinical suspicion was very helpful in diagnosis.

Out of 50 cases 1 false positive diagnosis of ectopic pregnancy was made. This patient had early intrauterine pregnancy that was not detected sonographically and associated corpus luteum cyst was misdiagnosed as an ectopic pregnancy. So transabdominal ultrasonography may miss a significant number of ectopic pregnancy cases. $8.7 \%$ of proven ectopics are sonographically normal ${ }^{13}$.

Analysis of ultrasonographic findings showed that individual finding like adnexal mass, culde-sac fluid or bulky uterus was less sensitive and specific in diagnosis of ectopic pregnancy. But their combined presence in ultrasonography increased the sensitivity and specificity in diagnosis of ectopic pregnancy.

In this study, $36(72 \%)$ cases were diagnosed as ectopic pregnancy by transabdominal ultrasound examination. Among them 35 cases correlated with surgical diagnosis. Remaining $14(28 \%)$ had diagnosis other than ectopic 
pregnancy by ultrasound examination, 9 of them were finally proved as ectopic pregnancy.

It was evident from the present study that transabdominal ultrasound examination diagnosed ectopic pregnancy with a sensitivity of $79.5 \%$ and specificity of $83.3 \%$. Predictive accuracy was $80 \%$. Other investigators such as Lawson ${ }^{9}$ and Levi $^{14}$ had almost similar diagnostic accuracy.

\section{Conclusion:}

Present study shows $80 \%$ accuracy of transabdominal ultrasonography in detecting ectopic pregnancy.Transabdominal ultrasonography is also helpful in differentiating pregnancy from many other simulating conditions like abortion, blighted ovum and molar pregnancy and thus reduces unnecessary laparotomy. so it can be used as a primary imaging modality in evaluating suspected ectopic pregnancy patient. The number of false positive and false negative diagnoses continues to be high. Pelvic inflammatory disease was the main mimicker, followed by normal pelvis and appendicitis. The researchers recommended further studies using a combination of transvaginal ultrasonogram and "discriminatory zone" of beta hCG to reduce the number of false negative rate in this study.

\section{References:}

1. Speroff L, Glass RH, Kase NG. Ectopic pregnancy. In: Speroff L, Glass RH, Kase NG, eds. Clinical Gynecologic Endocrinology and Infertility. USA: Williams and Wilkins; 1999.

2. Goldner TE, Lawson HW, Xia Z, Atrash HK. Surveillance for ectopic pregnancy. United States, 1970-1989. MMWR Morb Mortal Wkly Rep. 1993; 42: $73-85$.
3. Centers for Disease Control. Current trends. Ectopic pregnancy - United States 1990-1992. MMWR Morb Mortal Wkly Rep. 1995; 44: 46-8.

4. Kadar N, Caldwell BV, Romero R. A method of screening for ectopic pregnancy and its indications. Obstet Gynaecol 1981; 58: 162-5.

5. Nyberg DA, Mack LA, Jeffery RB, Laing FC. Endovaginal sonographic evaluation of ectopic pregnancy: a prospective study. AJR 1987; 149: 1181-6.

6 Reece EA, Petric RH, Sirmans MF, Finster M, Todd WD. Combined intrauterine and extrauterine gestations: review. Am J Obstet Gynecol 1983; 146(5): 323-327.

7. Chambers SE, Muir BB, Haddad NG. Ultrasound evaluation of ectopic pregnancy including correlation with human chorionic gonadotrophin levels. Br J Radiol 1990; 63: 246-50.

8. Cacciatore B, Stenman UH, Ylöstalo V, Carwright PS. Comparison of abdominal and vaginal sonography in suspected ectopic pregnancy. Obstet Gynecol 1989; 73(5): 770-4.

9. Lawson TL. Ectopic pregnancy: Criteria and accuracy of ultrasound diagnosis. AJR 1978; 131: 153-6.

10. Frates MC, Laing FC. Sonographic evaluation of ectopic pregnancy: an update. AJR 1995; 165: 251-9.

11. Nyberg DA, Hughes MP, Mack LA, Wang KY. Extrauterine findings of ectopic pregnancy at transvaginal US: Importance of echogenic fluid. Radiology 1992; 183: 407-11.

12. Mathony BS, Filly RA, Nyberg DA, Callen PW. Sonographic evaluation of ectopic pregnancy. J Ultrasound Med 1985; 4: 221-4.

13. Lyonf EA, Levi CS \& 